\title{
Fading Channels Parametric Data Simulation Supported by Real Data from Outdoor Experiments
}

\author{
Azra Kapetanovic, Mohamed A. Zohdy, Redhwan Mawari \\ Department of Electrical and Computer Engineering, Oakland University, Rochester, MI, USA \\ Email: akapetan@oakland.edu, zohdyma@oakland.edu,rmawari@oakaland.edu
}

How to cite this paper: Kapetanovic, A., Zohdy, M.A. and Mawari, R. (2017) Fading Channels Parametric Data Simulation Supported by Real Data from Outdoor Experiments. Journal of Signal and Information Processing, 8, 113-131.

https://doi.org/10.4236/jsip.2017.83008

Received: April 2, 2017

Accepted: July 16, 2017

Published: July 19, 2017

Copyright (c) 2017 by authors and Scientific Research Publishing Inc. This work is licensed under the Creative Commons Attribution International License (CC BY 4.0).

http://creativecommons.org/licenses/by/4.0/

\begin{abstract}
Optimizing the estimates of received power signals is important as it can improve the process of transferring an active call from one base station in a cellular network to another base station without any interruptions to the call. The lack of effective techniques for estimation of shadow power in fading mobile wireless communication channels motivated the use of Kalman Filtering (KF) as an effective alternative. In our research, linear second-order state space Kalman Filtering was further investigated and tested for applicability. We first created simulation models for two KF-based estimators designed to estimate local mean (shadow) power in mobile communications corrupted by multipath noise. Simulations were used extensively in the initial stage of this research to validate the proposed method. The next challenge was to determine if the models would work with real data. Therefore, in [1] we presented a new technique to experimentally characterize the wireless small-scale fading channel taking into consideration real environmental conditions. The twodimensional measurement technique enabled us to perform indoor experiments and collect real data. Measurements from these experiments were then used to validate simulation models for both estimators. Based on the indoor experiments, we presented new results in [2], where we concluded that the second-order KF-based estimator is more accurate in predicting local shadow power profiles than the first-order KF-based estimator, even in channels with imposed non-Gaussian measurement noise. In the present paper, we extend experiments to the outdoor environment to include higher speeds, larger distances, and distant large objects, such as tall buildings. Comparison was performed to see if the system is able to operate without a failure under a variety of conditions, which demonstrates model robustness and further investigates the effectiveness of this method in optimization of the received signals. Outdoor experimental results are provided. Findings demonstrate that the second-order Kalman filter outperforms the first-order Kalman filter.
\end{abstract}




\section{Keywords}

Kalman Filtering, Small-Scale Fading, Large-Scale Fading, Shadowing, Signal Estimation

\section{Introduction}

Because wireless technology and smart cell phones are experiencing dramatic growth, the accurate estimation of local mean (shadow) power in a cell phone is becoming a popular area of challenge for engineers in both industry and academia. Researchers are encouraged to find ways to enhance device performance in power control and handoff, particularly to address mobility-induced fading in metropolitan areas.

Wireless cell phones operate by transferring information over a distance between two or more stations that are not connected by cables. Instead, cell phones use radio waves to carry information, such as sound, by systematically modulating some property of electromagnetic energy waves transmitted through space, such as their amplitude, frequency, phase, or pulse width [3]. A transmitted signal from a cell tower will undergo changes while traveling through the propagation path to the cell phone. These changes may fluctuate with time, geographical position, and radio frequency. The term fading is used to describe the effect of these changes. As a result, the quality of communications decreases.

Two significant forms of fading in cellular communications are multipath and shadow fading. Since cell phone users tend to move a lot, received signal strength fluctuates with these two multiplicative forms of fading [4]. In the outdoor environment, shadow fading in cell phones causes long-term variation primarily caused by nearby mountains or tall buildings. Tall building structures shadow the radio signal, which results in a power drop at a receiver input. Multipath, as illustrated in Figure 1, results in fluctuations of the signal amplitude because of the addition of signals that arrive with different phases [2].

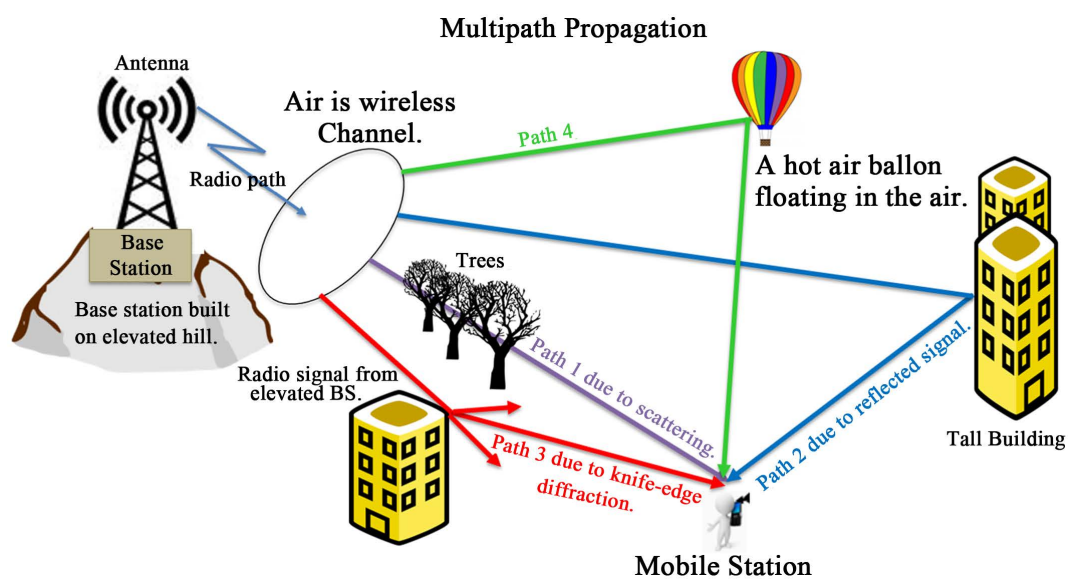

Figure 1. Versions of the same signal can take many different paths between cell tower and cell phone which causes fading effect in the resultant received signal. 
Having an accurate estimate of the shadowing component of a received power signal will allow the mobile communication system to efficiently compensate for the signal degradation that will occur. As a result, it can help the system perform handoff at the most effective times (predict when and where to handoff user). In [5], the authors have presented and discussed the limitation of different types of windows-based estimators that are utilized to filter multipath noise from the instantaneous received power signal to estimate the local mean shadow power. Unfortunately, window-based estimators work well only under the assumption that the shadowing component is relatively constant during the window period. However, shadowing components are not constant; the fluctuations can vary and at times can significantly decrease the performance of the windows-based estimate. In [2], we proposed a second-order KF-based estimator as an alternative method to windows-based estimation for estimating local mean (shadow) power.

Kalman filtering is a very effective algorithm that uses a series of measured observations and produces optimal estimates of states as explained in [6] [7] [8]. We applied this method to derive equations for an estimator that can estimate local mean (shadow) power profiles. In the initial stage of research, we used simulation models to validate the proposed method. The next challenge was to see if the model would work with real data. Therefore, in [1] we presented a new technique to experimentally characterize the wireless small-scale fading channel, taking into consideration real environmental conditions. This new two dimensional measurement technique provided essential information regarding the constructive and destructive interference patterns caused by the interaction between the mobile station (while in motion) and surrounding obstacles. The two dimensional measurement technique enabled us to perform indoor experiments and collect real data, which we then used to confirm validation of the simulation model for the second-order KF-based estimator.Based on results from the indoor experiments, we concluded that the second-order KF-based estimator is more accurate in predicting local mean (shadow) power profiles than the first-order KF-based estimator, even in channels with imposed non-Gaussian measurement noise.

The next challenge was to find a way to measure cell phone signal strength outside of the lab environment and to test our second-order KF-based estimator. In this paper, we looked at mobility-induced fading and present experimental results from the outdoor environment that further confirmed validation of the proposed method. We will explain how Kalman filter method can be applied in optimization of received signals in mobile communications. The system was able to operate without a failure under a variety of conditions, which demonstrates model robustness. In subsequent sections, it will be demonstrated that the second-order KF based estimator we designed exceeds the performance of the first-order KF-based estimator, even in the outdoor environment where parameters for mobile velocity varied. 


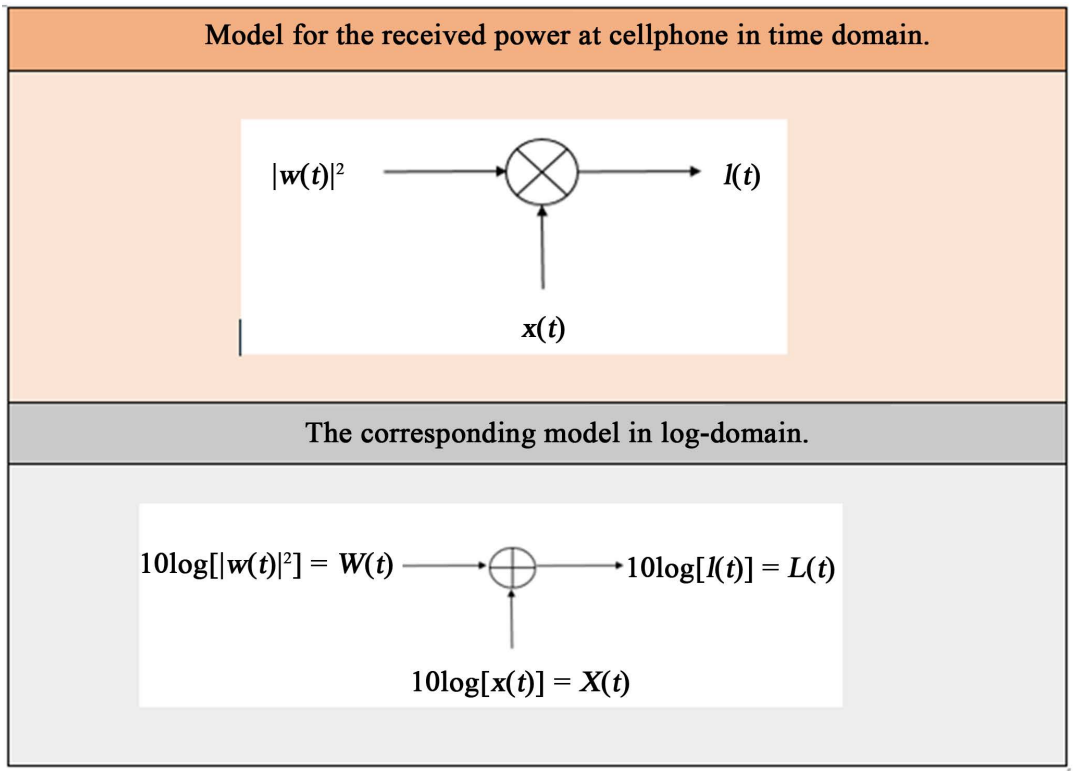

Figure 2. Model for the received power at cell phone.

\section{Model for Multipath Signal}

The description of Shadow Power Signal and its models as they pertain to our problem are presented in this section. In a wireless cellular radio environment, a model for an instantaneous received power signal $l(t)$ at a cell phone is given in Figure 2, where $|w(t)|^{2}$ represents fast power fluctuation due to multipath and $x(t)$ represents slow power fluctuation due to shadowing. Many common shadow power estimation methods in industry rely on an accurate model for multipath. Multipath is often modeled as Rayleigh noise for modeling purposes. It is customary to express power measurements in decibels. Handoff algorithms rely on estimates of shadow power in decibels [9].

To solve the problem, we start with the multipath model shown in Equation (2).

$$
\begin{gathered}
w(R)=\frac{1}{R}\left[a_{1} \mathrm{e}^{j\left(\omega_{D} \cos \left(\theta_{1}\right) t+\phi_{1}\right)}+a_{2} \mathrm{e}^{j\left(\omega_{D} \cos \left(\theta_{2}\right) t+\phi_{2}\right)}+a_{R} \mathrm{e}^{j\left(\omega_{D} \cos \left(\theta_{R}\right) t+\phi_{R}\right)}\right] \\
w(R+1)=\frac{1}{R+1}\left[w(R) * R+a_{R+1} \mathrm{e}^{j\left(\omega_{D} \cos \left(\theta_{R+1}\right) t+\phi_{R+1}\right)}\right]
\end{gathered}
$$

where:

- $v$ is the magnitude of the mobile velocity $[10 \mathrm{~m} / \mathrm{s}-30 \mathrm{~m} / \mathrm{s}]$,

- $\lambda$ is the wavelength corresponding to the carrier frequency, which is typically $\frac{3 \times 10^{8}}{700 \mathrm{MHz}}=0.42 \mathrm{~Hz}$,

- $\omega_{D}$ is the Doppler spread equal to $\frac{2 \pi v}{\lambda}$. Range used here is $\left[\frac{2 * \pi * 10 \mathrm{~m} / \mathrm{s}}{\frac{3}{7} \mathrm{~Hz}}\right.$ $\left.\frac{2 * \pi * 30 \mathrm{~m} / \mathrm{s}}{\frac{3}{7} \mathrm{~Hz}}\right]$

- $R$ is the number of paths in the multipath power [20 - 30], 
- $a_{r}$ is gain [0 - 20],

- $\theta_{r}$ represents angles between incoming waves and mobile antenna. The value range is uniformly distributed $[-\pi, \pi]$. Other distributions like Normal can be used,

- $\left\{\phi_{r}\right\}_{r=1}^{R}$ represents phase random variables whose values are also uniformly distributed $[-\pi, \pi]$.

\section{Model for Shadow Power Signal}

Equation (3) shows a widely accepted first-order state space model for the shadow process given by [10]. We derived a second-order state space model for the shadow process as shown in Equation (4).

$$
\begin{gathered}
x_{k}=a_{1} x_{k-1}+\phi_{k} \\
x_{k}=a_{2} x_{k-2}+a_{1} x_{k-1}+\phi_{k}
\end{gathered}
$$

First shadow power coefficient $a_{1}$ is given by Equation (5) and second shadow power coefficient $a_{2}$ is given by Equation (6):

$$
\begin{aligned}
& a_{1}=\mathrm{e}^{\frac{-v T_{s}}{X_{c 1}}} \\
& a_{2}=\mathrm{e}^{\frac{-v T_{s}}{X_{c 2}}}
\end{aligned}
$$

where, $v$ is the magnitude of the mobile velocity, $T_{s}$ is time sample, and $X_{c}$ is effective correlation distance. The effective correlation distance is key attribute of the wireless environment. In urban area it can be as low as $10 \mathrm{~m}$ while in suburban areas it can be as high as $500 \mathrm{~m}$. The effective correlation distance is given in Equation (7), where variable $D$ is the distance between cell tower and cell phone measured in meters. Term $\varepsilon_{D}$ is the correlation coefficient of shadow process between two points separated by distance $\mathrm{D}$.

$$
X_{c}=\frac{-D}{\ln \left(\varepsilon_{D}\right)}
$$

Finally, system noise covariance is given in Equation (8), where term $\sigma_{s}^{2}$ denotes the shadow variance which depends on environment. In urban areas, typical value for shadow variance is $4 \mathrm{~dB}$ while in suburban areas typical value is 8 dB. Term $\phi_{k}$ in Equations (3) and (4) denotes zero mean white Gaussian noise with variance $\sigma_{\phi}^{2}$.

$$
\sigma_{\phi}^{2}=\left(1-a^{2}\right) * \sigma_{s}^{2}
$$

\section{Kalman Filter Algorithm}

Kalman Filter theory was developed and introduced in 1960 by Dr. Rudolf Kalman. This led to the use of the Kalman filter during the Apollo program, carried out by NASA, which accomplished landing the first humans on the Moon. Since then, his contributions and thoughts educated and inspired inventors across many disciplines. As a result, the Kalman filter has been the subject of extensive research and application, especially in digital computing. 
In this research, we applied the Kalman filter algorithm to estimate power signal in a mobile communication corrupted by multipath noise. The Kalman filter is a form of a linear algorithm for optimal recursive estimation of a system state with a specific set of output equations. The estimates are calculated every time a new measurement is received. Data received is processed sequentially, so it is not necessary to store the complete data set or to reprocess existing data when new measurement data is received.

\subsection{Derivation of the Discrete-Time Linear Kalman Filter}

This section derives the equations of the discrete-time Kalman filter. This filter is applied as a recursive solution to the estimation problem studied in this research. To use the Kalman filter to estimate signal of interest, one must first create matrices to fix the system model into a Kalman filter format. The following sets of equations describe the format of the linear discrete-time system:

$$
\begin{gathered}
x_{k}=A_{k-1} x_{k-1}+B_{k-1} u_{k-1}+w_{k-1} \\
y_{k}=H_{k} x_{k}+v_{k}
\end{gathered}
$$

The Kalman filter is a great tool, but its computation is complex and requires some explanation. An optimal value for $x_{k}$ in Equation (9) is calculated based on the available knowledge of the system dynamics and the noise measurement $y_{k}$. In Equation (10), $y_{k}$ represents the measured output of the system (measurement of system state) with measurement noise.

The k's on the subscripts are states and can be treated as discrete time intervals. In general, when applying the Kalman Filter, the goal is to estimate state $x_{k}$. For example, in signal processing, it is basically the estimate of some signal $\boldsymbol{x}$ that we want to find for each subsequent $k$. During this process, the Kalman filter forms ana priori estimate and an a posterior estimate denoted as $\hat{x}_{k}^{-}$and $\hat{x}_{k}^{+}$. Equation (11) computes the expected value of $x_{k}$ conditioned on all of the measurement up to time k. Similarly, Equation (12) computes the expected value of $x_{k}$ conditioned on all of the measurements after time $k$.

$$
\begin{aligned}
\hat{x}_{k}^{-} & =E\left[x_{k} \mid y_{1}, y_{2}, y_{3}, \cdots, y_{k-1}\right] \\
& =\text { estimate of } x_{k} \text { before the measurement at time } k \text { is proccessed } \\
\hat{x}_{k}^{+} & =E\left[x_{k} \mid y_{1}, y_{2}, y_{3} \ldots y_{k}\right] \\
& =\text { estimate of } x_{k} \text { after the measurement at time } k \text { is proccessed }
\end{aligned}
$$

Each system has to have initial values. Notation $\hat{x}_{0}^{+}$denotes an initial estimate of $x_{0}$ before any measurements are taken. The first measurement in this algorithm is taken at time $k=1$. During this time period, the system does not have any measurements available to estimate $x_{0}$, and therefore $\hat{x}_{0}^{+}$is formed as the best expected value of the initial state $x_{0}$

$$
\hat{x}_{0}^{+}=E\left(x_{0}\right)
$$

This algorithm takes into account the measurement noise, process noise, and the previous estimated output values so that it can minimize the prediction error upon a continuous cycle of prediction and filtering. It looks at the error between 
the true state and the estimated state. Therefore, the next step is to derive an error equation. Equations (14) and (15) define an priori estimate error and an posteriori estimate error, respectively.

$$
\begin{aligned}
& e_{k}^{-}=x_{k}-\hat{x}_{k}^{-} \\
& e_{k}^{+}=x_{k}-\hat{x}_{k}^{+}
\end{aligned}
$$

Then, Equations (14) and (15) are used to compute covariance of the estimation error, which is denoted as $P_{k}$. The term $P_{k}^{-}$denotes the covariance of the estimation error of $\hat{x}_{k}^{-}$, and $P_{k}^{+}$denotes the covariance of the estimation error of $\hat{x}_{k}^{+}$:

$$
\begin{aligned}
& P_{k}^{-}=E\left[\left(x_{k}-\hat{x}_{k}^{-}\right)\left(x_{k}-\hat{x}_{k}^{-}\right)^{\mathrm{T}}\right] \\
& P_{k}^{+}=E\left[\left(x_{k}-\hat{x}_{k}^{+}\right)\left(x_{k}-\hat{x}_{k}^{+}\right)\right]^{\mathrm{T}}
\end{aligned}
$$

After the measurement at time $k-1$ is processed, the estimate of the $x_{k-1}$ is computed, which is denoted as $\hat{x}_{k-1}^{+}$. Also, the covariance of that estimate is computed at the same time, and it is denoted as $P_{k-1}^{+}$. Then, at time $\mathrm{k}$, before the measurement is processed, estimate of $x_{k}$ is computed and denoted as $\hat{x}_{k}^{-}$ Then, the covariance of these estimates are computed and denoted as $P_{k}^{-}$Then, at time $k=1$, the measurement is processed to refine the estimate of $x_{k}$. The resulting improved estimates of $x_{k}$ and its covariance are denoted as $\hat{x}_{k}^{+}$and $P_{k}^{+}$.

To begin the estimation process, initial values of the system determined in Equation (13) must be initialized. Then, with $\hat{x}_{0}^{+}$, variable $\hat{x}_{1}^{-}$is computed using Equation (18). Based on this equation, the time update equation for $\hat{x}$ is computed as indicated in Equation (19).

$$
\begin{gathered}
\hat{x}_{1}^{-}=A_{0} \hat{x}_{0}^{+}+B_{0} u_{0} \\
\hat{x}_{k}^{-}=A_{k-1} \hat{x}_{k-1}^{+}+B_{k-1} u_{k-1}
\end{gathered}
$$

From time $(k-1)^{+}$to time $(k)^{-}$, the state estimate and its mean propagates the same way. As there are no additional measurements available between these two time-steps, the state estimate has to be updated based on a knowledge of system dynamics.

Next step is to derive time-update equation for the covariance of the state estimation error. The term $P_{0}^{+}$represents the covariance of the initial estimate of $x_{0}$ (the uncertainty in initial estimates of $x_{0}$ ). If the exact value of the initial state is known, then $P_{0}^{+}$can be set to zero. However, if the initial value is not known, then then $P_{0}^{+}=\infty I$. The mathematical from for $P_{0}^{+}$is shown in Equation (20). The general description of how the covariance of the state of a linear discrete-time system propagates with time is given by Equation (21). The term $P_{1}^{-}$can be computed by substituting $P_{0}^{+}$value from Equation (20). The time equation for term $P$ in general form is given by Equation (23).

$$
P_{0}^{+}=E\left[\left(x_{0}-\hat{x}_{0}^{+}\right)\left(x_{0}-\hat{x}_{0}^{+}\right)\right]^{\mathrm{T}}
$$




$$
\begin{gathered}
P_{k}=A_{k-1} P_{k-1} A_{k-1}^{\mathrm{T}}+Q_{k-1} \\
P_{1}^{-}=A_{0} P_{0}^{+} F_{0}^{\mathrm{T}}+Q_{0} \\
P_{k}^{-}=A_{k-1} P_{k-1}^{+} A_{k-1}^{\mathrm{T}}+Q_{k-1}
\end{gathered}
$$

Final step requires derivation of measurement-update equations for $\hat{x}$ and $P$. Given $\hat{x}_{k}^{-}$from Equation (19), we need to find a method to compute $\hat{x}_{k}^{+}$, the estimate of $x_{k}$, which takes the measurement $y_{k}$ into account. Recall $y_{k}$ in Equation (10) represents the measurement of the system state with measurement noise $v_{k}$. Measurement noise is usually caused by the measurement instrument. Based on the recursive least square estimation theory, we know that the availability of $y_{k}$ changes the estimate of a constants $x$ as follows:

$$
\begin{aligned}
K_{k} & =P_{k-1} H_{k}^{\mathrm{T}}\left(H_{k} P_{k-1} H_{k}^{\mathrm{T}}+R_{k}\right)^{-1} \\
\hat{x}_{k} & =\hat{x}_{k-1}+K_{k}\left(y_{k}-H_{k} \hat{x}_{k-1}\right) \\
P_{k} & =\left(I-K_{k} H_{k}\right) P_{k-1}\left(I-K_{k} H_{k}\right)^{\mathrm{T}}+K_{k} R_{k} K_{k}^{\mathrm{T}} \\
& =\left(P_{k-1}^{-1}+H_{k}^{\mathrm{T}} R_{k}^{-1} H_{k}\right)^{-1} \\
& =\left(I-K_{k} H_{k}\right) P_{k-1}
\end{aligned}
$$

where $\hat{x}_{k-1}$ and $P_{k-1}$ are estimates before the measurement is processed, while $\hat{x}_{k}$ and $P_{k}$ take the measurements $y_{k}$ into account. The next step is to rewrite Equations (24) through (26) in a format that Kalman used when he derived his estimation theory. To formulate the measurement-update equations for $\hat{x}_{k}$ and $P_{k}$, simply perform the following substitutions: substitute $\hat{x}_{k-1}$ with $\hat{x}_{k}^{-}$, $P_{k-1}$ with $P_{k}^{-}, \hat{x}_{k}$ with $\hat{x}_{k}^{+}$, and $P_{k}$ with $P_{k}^{+}$. These substitutions lead to the following equations:

$$
\begin{aligned}
K_{k} & =P_{k}^{-} H_{k}^{\mathrm{T}}\left(H_{k} P_{k}^{-} H_{k}^{\mathrm{T}}+R_{k}\right)^{-1} \\
\hat{x}_{k}^{+} & =\hat{x}_{k}^{-}+K_{k}\left(y_{k}-H_{k} \hat{x}_{k}^{-}\right) \\
P_{k}^{+} & =\left(I-K_{k} H_{k}\right) P_{k}^{-}\left(I-K_{k} H_{k}\right)^{\mathrm{T}}+K_{k} R_{k} K_{k}^{\mathrm{T}} \\
& =\left[\left(P_{k}^{-}\right)^{-1}+H_{k}^{\mathrm{T}} R_{k}^{-1} H_{k}\right]^{-1} \\
& =\left(I-K_{k} H_{k}\right) P_{k}^{-}
\end{aligned}
$$

The matrix $K_{k}$ given in Equation (27) is called the Kalman filter gain. This gain is a blending factor that minimizes the a posteriori error covariance. If the $x_{k}$ is a constant, then $A_{k}=I, Q_{k}=0$, and $u_{k}=0$.

The random variable $w_{k}$ represents process noise and $v_{k}$ represents measurement noise. Terms $v_{k}$ and $w_{k}$ are independent of each other. They are assumed to be white and with normal probability distributions

$$
\begin{gathered}
p(w) \sim N\left(0, Q_{k}\right) \\
p(v) \sim N\left(0, R_{k}\right)
\end{gathered}
$$

In outdoor experiments, the process noise covariance matrix $Q_{k}$ and measurement noise covariance matrix $R_{k}$ can change for every measurement. 


\subsection{First-Order Kalman Filter Application to Fading Channels}

The Kalman Filter is a form of a linear algorithm for optimal recursive estimation of a system state with a specific set of output equations. To build a simulator, understanding of system model and its dynamic behaviors is necessary. Then, the system must be represented in the state space format to be able to apply Kalman filtering. In other words, we need to mathematically model its states and parameters. This section presents set of equations used to create first-order KF-based estimator. References [10] and [11] were useful for programming in MATLAB during the initial stages of research.

To build an estimation model in MATLAB, we started with equations introduced in Section 4.1 and substituted suitable entries from this problem to reflect the linear channel model [12]. Equations (9) and (10) can be rewritten as Equations (32) and (33). This assumes a linear time invariant system with a mean of zero and white noise on both the state and output.

$$
\begin{gathered}
x_{k}=A x_{k-1}+B u_{k-1}+w_{k-1} \\
l_{k}=H x_{k}+v_{k}
\end{gathered}
$$

where:

- $x_{k}$ is a symbol value of shadow signal state that needs to be estimated.

- $u_{k}$ is the control signal for handoff.

- $w_{k}$ is process white noise.

- $v_{k}$ is measurement noise.

- $l_{k}$ is the measurement value for both shadow and multipath. In this document, $l_{k}$ or $L(k)$ is the measurement value used to update shadow power estimate.

After initializing Kalman filter using initial values $x_{O}$ and $P_{O}$ as explained in Section 4.1, time-update and measurement-update equations had to be identified. These equations are computed for each time step $k=1,2,3$. Equations (34) and (35) represent the "Time Update" state of the Kalman Filter, also known as the "Prediction States." Equations (34) and (35) are derived by substituting suitable entries from this problem into Equations (19) and (22). For first-order KF, matrix A was set to $a_{1}$, which represents the first shadow power coefficient given by Equation (5.5). Term for system noise covariance $Q_{k}$ in Equation (22), was substituted with $\sigma_{\phi}^{2}$ in Equation (35) and it denotes variance defined in Equation (34). Here we project the current state estimate forward in time with Equation (34) projecting the state ahead and Equation (35) projecting the error covariance ahead as represented below:

$$
\begin{gathered}
\hat{x}_{k}^{-}=a_{1} \hat{x}_{k-1}^{+} \\
P_{k}^{-}=a_{1} P_{k-1}^{+}+\sigma_{\phi}^{2}
\end{gathered}
$$

where $\hat{x}_{k}^{-}$is the rough estimate before the measurement $l_{k}$ is processed at time $k$.

Similarly, Equations (37), (38) and (39) belong to the "Measurement Update" state of the linear Kalman Filter, also known as the "Correction State." Equations 
(37), (38) and (39) are derived by substituting suitable entries from this problem into Equations (27), (28) and (29). Here we adjust the projected estimate by an actual measurement at time $k$. Equation (37) was derived by substituting environment noise covariance $R_{k}$ with $\sigma_{H}^{2}$ defined in Equation (36).

$$
\sigma_{H}^{2}=\frac{\pi^{2}}{6}[10 / \ln 10]^{2}
$$

Equation (37) computes the Kalman Gain, Equation (38) adjusts the projected estimate by an actual measurement $l_{k}$, and Equation (39) updates the error covariance, as follows:

$$
K_{k}=P_{k}^{-}\left(P_{k}^{-}+\sigma_{H}^{2}\right)^{-1} \text {. }
$$

If $R$ is small and $P_{k}^{-}$is close to Identity, then $K_{k}=H^{\mathrm{T}}\left(H H^{\mathrm{T}}\right)^{-1}$, which is a well-known Pseudo inverse.

$\hat{x}_{k}$ : Estimate of $x$ after the actual measurement $l_{k}$ at time $k$.

$$
\begin{gathered}
\hat{x}_{k}=\hat{x}_{k}^{-}+K_{k}\left(l_{k}-\hat{x}_{k}^{-}\right) \\
P_{k}^{+}=\left(1-K_{k}\right) P_{k}^{-}
\end{gathered}
$$

The next step is to represent these estimates over a period of sufficient time. The output estimate in the previous step will be the input estimate in the next step. The main goal is to find an optimal value for $\hat{x}_{k}$.

\subsection{Second-Order Kalman Filter Application to Fading Channels}

In this research, we assumed that the first-order state space model can be used to model Shadow Power. To extend the first-order state space model equations [1] presented in Section 4.2, a second-order state space model for the linear Kalman Filter was formulated and applied as suggested in the equations below. With this notation, we can describe an algorithm for the second-order KF as follows:

- $a_{1}$ is the first Shadow power coefficient as defined in Equation (5).

- $a_{2}$ is the second Shadow power coefficient as defined in Equation (6).

- $X_{c}$ is the effective correlation distance as defined in Equation (7).

- $\varepsilon_{D}$ is the correlation coefficient of the shadow process between two points separated by a distance $D$ as measured in meters,

$$
x_{k}=\left[\begin{array}{c}
x_{1 k} \\
x_{2 k}
\end{array}\right] .
$$

Equation (40) shows $x_{k}$ expressed in matrix format for second-order state space.

Next set of equations present prediction states for second-order linear Kalman filter. Equation (34) can be rewritten as Equation (41). Therefore, Equation (41) in this section projects the state ahead, and Equation (43) projects the error covariance ahead. For the second-order KF, the matrices are defined as follows:

$$
A=\left[\begin{array}{cc}
1 & 0 \\
a_{1 k} & a_{2 k}
\end{array}\right], \quad B=\left[\begin{array}{l}
0 \\
0
\end{array}\right], \quad H=\left[\begin{array}{ll}
0 & 1
\end{array}\right]
$$




$$
\left[\begin{array}{c}
\hat{x}_{1 k}^{-} \\
\hat{x}_{2 k}^{-}
\end{array}\right]=\left[\begin{array}{cc}
1 & 0 \\
a_{1 k} & a_{2 k}
\end{array}\right]\left[\begin{array}{l}
\hat{x}_{1 k-1} \\
\hat{x}_{2 k-2}
\end{array}\right]+\left[\begin{array}{l}
0 \\
0
\end{array}\right] u_{k} .
$$

$B$ is input matrix that relates the control input to the state $x_{k}$. Matrix $H$ is output equation whose function is to relate state to the measured output $l_{k}$.

Parameter Q in Equation (42) represents the predicted process noise. Term $\sigma_{s}$ denotes the shadow variance with range from $4 \mathrm{~dB}$ to $8 \mathrm{~dB}$. The notation $\left(1-a^{2}\right) * \sigma_{S}^{2}$ in Equation (42) denotes the variance of the zero mean white Gaussian noise.

$$
Q=\left[\begin{array}{cc}
\left(1-a_{1}^{2}\right) \sigma_{s}^{2} & \left(1-a_{2} a_{1}\right) \sigma_{s}^{2} \\
\left(1-a_{2} a_{1}\right) \sigma_{s}^{2} & \left(1-a_{2}^{2}\right) \sigma_{s}^{2}
\end{array}\right]
$$

Equation (43) can then be expressed in the following state space format:

$$
P_{k}^{-}=\left[\begin{array}{cc}
1 & 0 \\
a_{1 k} & a_{2 k}
\end{array}\right] P_{k-1}^{+}\left[\begin{array}{cc}
1 & a_{1 k} \\
0 & a_{2 k}
\end{array}\right]+\left[\begin{array}{cc}
\left(1-a_{1}^{2}\right) \sigma_{s}^{2} & \left(1-a_{2} a_{1}\right) \sigma_{s}^{2} \\
\left(1-a_{2} a_{1}\right) \sigma_{s}^{2} & \left(1-a_{2}^{2}\right) \sigma_{s}^{2}
\end{array}\right]
$$

Next set of equations present correction states. Equation (44) was used to compute Kalman gain, which takes into consideration measurement noise due to multipath.

$$
\begin{gathered}
H=\left[\begin{array}{ll}
0 & 1
\end{array}\right] \\
H^{\mathrm{T}}=\left[\begin{array}{l}
0 \\
1
\end{array}\right] \\
K_{k}=P_{k}^{-} H_{k}^{\mathrm{T}}\left(H_{k} P_{k}^{-} H_{k}^{\mathrm{T}}+\sigma_{H}^{2}\right)^{-1}
\end{gathered}
$$

Equation (45) updates the estimate via $l_{k}$, a measured value, and Equation (46) updates the error covariance.

$$
\begin{gathered}
{\left[\begin{array}{c}
\hat{x}_{1 k}^{+} \\
\hat{x}_{2 k}^{+}
\end{array}\right]=\left[\begin{array}{c}
\hat{x}_{1 k}^{-} \\
\hat{x}_{2 k}^{-}
\end{array}\right]+K_{k}\left(l_{k}-H\left[\begin{array}{c}
\hat{x}_{1 k}^{-} \\
\hat{x}_{2 k}^{-}
\end{array}\right]\right)} \\
P_{k}^{+}=\left(I-K_{k} H\right) P_{k}^{-}
\end{gathered}
$$

$X^{*}$ in Equation (47) is the optimal estimate of the second order shadow process.

$$
X^{*}=H\left[\begin{array}{l}
\hat{x}_{1 k} \\
\hat{x}_{2 k}
\end{array}\right]
$$

It is assumed that channel variation is mainly due to the changing mobile velocity and the correlation distance. Therefore, only the variation of the shadow process coefficient is considered. The smaller the sample period, the closer the shadow process coefficient is to one.

When the channel is nonlinear, the Unscented Kalman Filter also can be applied to the state space model optimize the shadow power presented in this section. In our problem, distribution of multipath is non-Gaussian. However, even when the white Gaussian noise assumption is not valid, the linear Kalman filter is still the optimal LMMSE estimator if the driving and measurement noises are white [5]. 


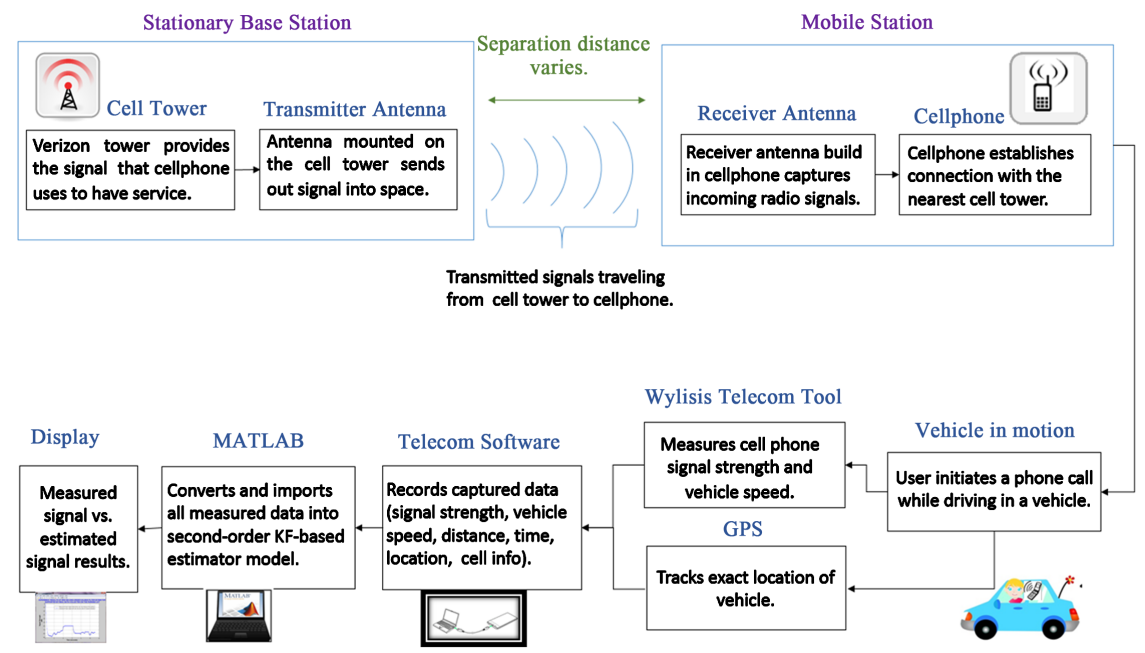

Figure 3. Block diagram of wireless communication system used in the outdoor experiment.

\section{Outdoor Experiments}

In [1], we presented a new technique to experimentally characterize the wireless small-scale fading channel, taking into consideration real environmental conditions. Then, using the technique that we described in [1], laboratory experiments were performed to collect real data and to validate the simulation model for the second-order KF-based estimator (designed to estimate power signal in cell phones). In [2], we showed that our simulation results in the MATLAB environment and laboratory experiment results validate the proposed algorithm and the theoretical analysis. We concluded that the second-order KF-based estimator is more accurate in predicting local shadow power profiles than the first-order KF-based estimator, even in channels with imposed non-Gaussian measurement noise. Next, we wanted to see how robust a second-order KF-based estimator is in the outdoor environment where we have different large-scale fading configurations. Therefore, outdoor experiments were set up as shown in Figure 3 to test a second-order KF-based model designed to estimate shadow power where wireless communication takes place while the user is in motion.

\subsection{Measurements}

A cell phone or portable phone uses radio waves to establish connection with its base station. Radio waves can travel long distances, but they easily get interrupted. As the transmitted signals travel from tower station to cell phone, they penetrate the atmosphere, and some signals are scattered, reflected, or observed. Objects obstructing the propagation path between the transmitter and receiver can cause variations in the received signal. All this can have a significant impact on signal strength in the cell phone device.

In this experiment, a mobile phone signal refers to signal strength received by a mobile antenna from a cellular network. There are several ways to measure mobile signal strength. The two most common units of measurement used in radio signals are dBm (decibels) and RSSI (Received Signal Strength Indicator). RSSI is 


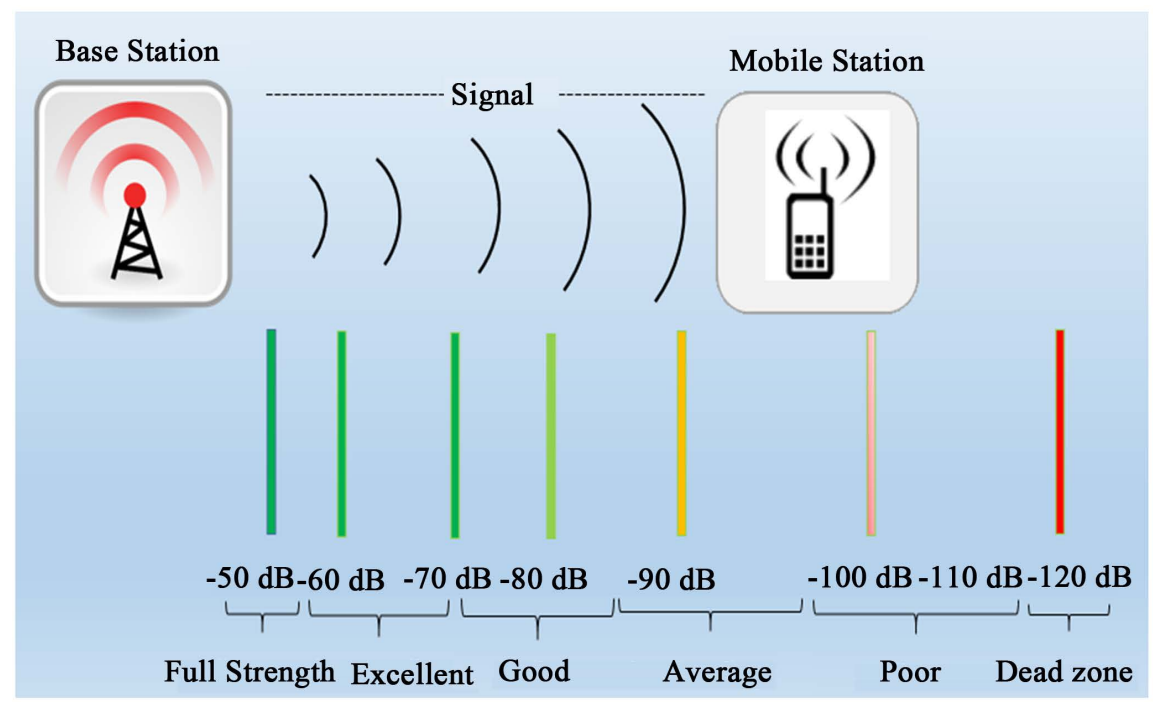

Figure 4. Cell phone signal strength range.

a measurement of the power present in a received radio signal. The higher the RSSI number, the stronger the signal. These values allow users to know when they are receiving a stronger signal or a weaker signal. Figure 4 shows that decibel values are expressed as negative numbers, which implies that the closer the value is to zero, the stronger the received signal. In Michigan, the standard frequency range for cellular phone operation is between $-50 \mathrm{~dB}$ to $-120 \mathrm{~dB}$. User will get the best signal at $-50 \mathrm{~dB}$ as it is considered full strength. On the other hand, $-120 \mathrm{~dB}$ is considered a dead zone and the user will have no phone service. It also indicates that an ideal signal strength for optimum performance of a cell phone is about $-65 \mathrm{dBm}$.

\subsection{Field Test Scenario}

Area or a region can impact signal strength or path loss. Therefore, as part of the experiment, we collected data in suburban and urban areas. Measurements have been conducted in two different environments while the user was driving a vehicle at different speeds:

1) Suburban environment, Oakland University campus in Auburn Hills, Michigan.

2) Urban environment, downtown Detroit, Michigan.

\subsection{Outdoor Experiment Prerequisites and Setup}

Valid research experiment must meet the certain criteria. To satisfy terrain requirement, an experiment must be conducted in an area that has good wireless cell phone coverage. Presence of large obstructing objects such as tall building structures are essential for forming a fading channel. Finally, equipment required for power signal data acquisition and processing include: base station, mobile station, vehicle, telecom tool, and laptop with built-in Bluetooth model and MATLAB software. 
Cell phones work by communicating via radio waves using a system of cell towers that send and receive calls. A base station, also known as a cell tower, is placed on a big metal pole about $300 \mathrm{ft}$. high. Cell towers have triangular platforms on the top of the pole for cellular providers to keep their equipment. The process of a cell phone tower transmission requires the following equipment: radios, antennas for receiving and transmitting radio frequency signals, computerized switching control equipment, GPS receivers, power sources, and some kind of protective cover. In this experiment, Verizon was the cellular provider and the location of the base station is shown in Figure 5.

A mobile station consists of the physical equipment (radio transceiver, display and digital signal processors) and software package needed for communication with a mobile network. In this experiment, the Samsung Galaxy S5 smartphone was used as a mobile station. Any type of legal vehicle is acceptable to perform a driving test on public roads. As cell phone user moves around while using a cell phone, tall buildings will shadow the radio signal, which can result in a power drop at the receiver input. In this research, initial experiments were performed next to large buildings on the Oakland University campus to create a shadow fading phenomena in the outdoor environment. Supplementary experiments were conducted in downtown Detroit.

Telecom tool that was released to the market by Wylisis in March 2017 is recommended for recording captured data (Figure 6) and vehicle movement. Telecom software has the capability to save logging data, which can be imported into MATLAB. Measured data includes cell tower location markers, signal strength, position, velocity, and time. Alternatively, the Data Acquisition Toolbox provides functions for connecting MATLAB to data acquisition hardware. Data can be analyzed as it is acquired or it can be saved for post-processing. Block diagram of wireless communication system used in the experiment setup outside of the lab environment is illustrated in Figure 3. The algorithm flow chart is presented in Figure 7.

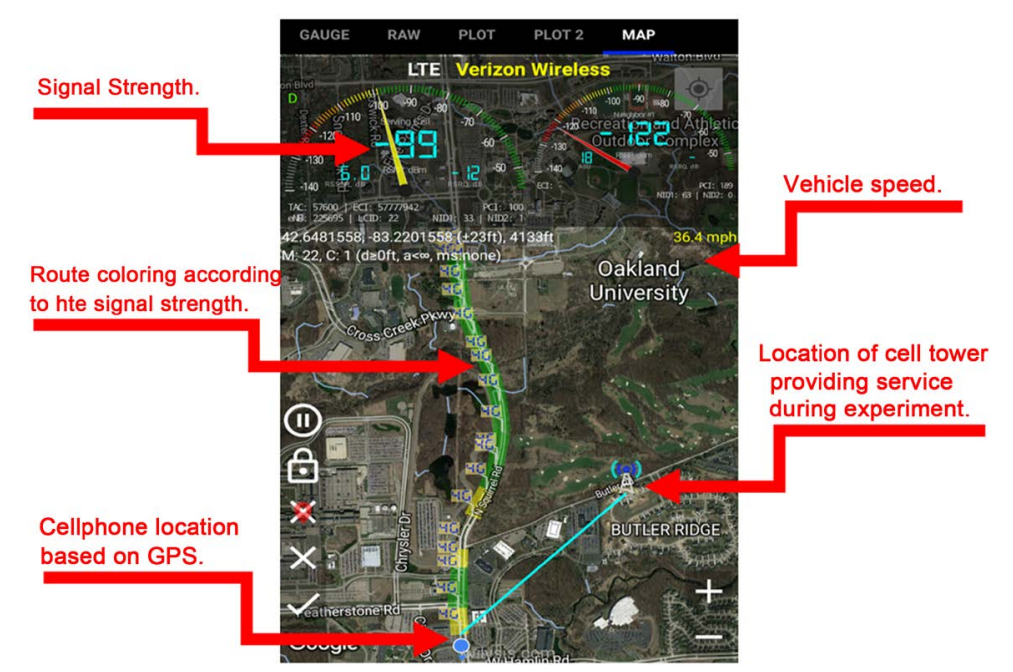

Figure 5. Showing distance between mobile station and base station during outdoor experiment at Oakland University. 


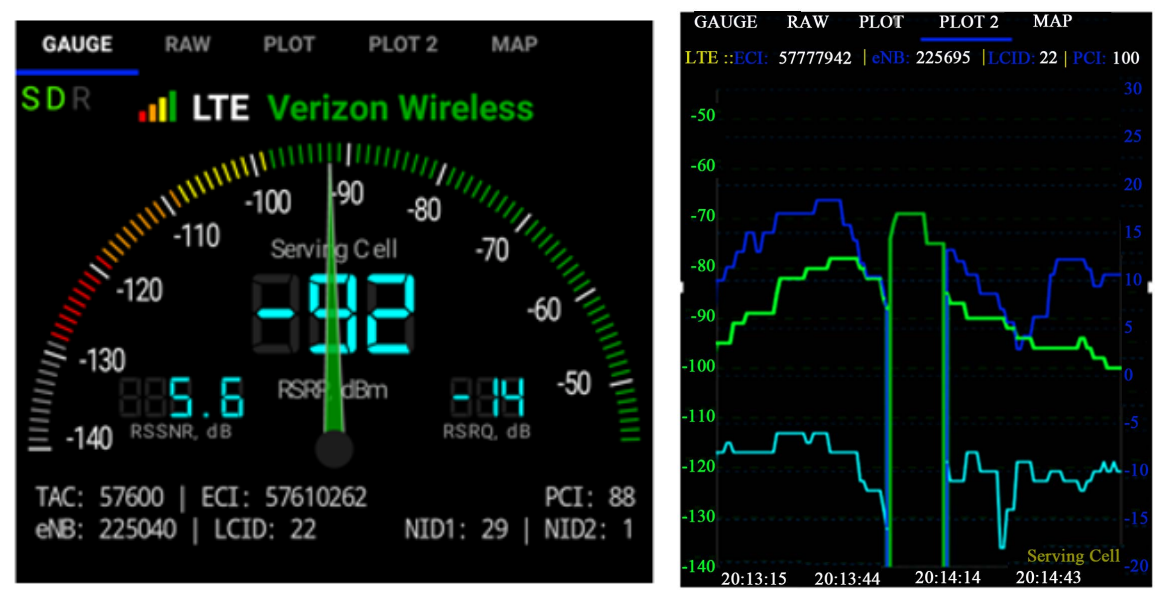

Figure 6. Signal Strength measurement during outdoor experiment.

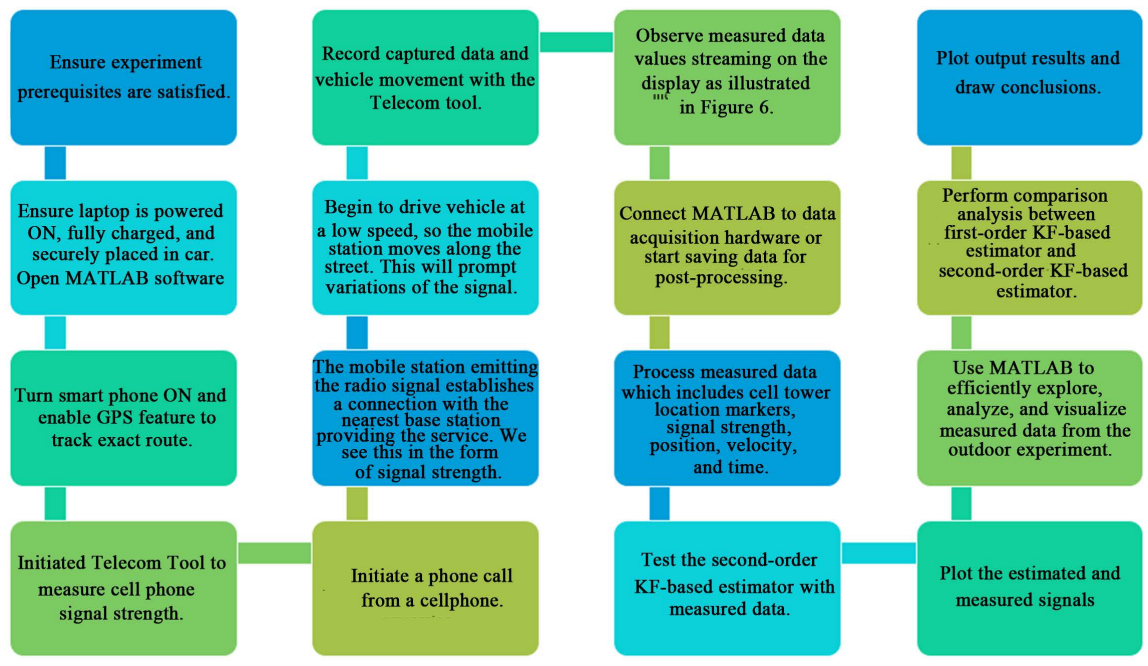

Figure 7. The algorithm flow chart.

\subsection{Outdoor Experiment Results}

This section presents outdoor experiment results for shadow process estimation and pertinent performance analysis. The purpose of these experiments was to study and analyze output results of the first-order state space model and to compare them to the second-order state space model while applying a Kalman Filter technique to determine shadow power signal in mobile communications from measurements that have impinged Rayleigh fast fading noise. As stated before, we were able to validate this concept through laboratory experiments with data from real scenarios, but those experiments performed in the indoor environment were limited by lower speed and obstacle contribution. The outdoor experiment allowed us to conduct tests that include higher mobile velocity, exact shadow variance values, and large-scale fading configurations.

Measurements have been conducted outside while the cell phone user was driving a vehicle at different speeds, which caused variation in default parameters, such as mobile receiver velocity, shadow variance, and effective correlation distance. Multiple experiment trials were performed to collect sufficient amount 
of data, but in this paper we include results from driving the vehicle at $36 \mathrm{mph}$ in urban area as shown in Figure 7. The second-order KF-based estimator performed equally well when we varied the parameters.

The plots of outdoor experiment results supported by the field data are shown in Figures 8-10. These plots show results of the actual shadow power signal and estimations with Kalman Filtering. In Figure 8 and Figure 9, predicted power signal with the second-order KF-based estimator (marked in blue color) is very close to the measured signal (marked in black color). However, Figure 10 shows noticeable disparity between measured signal (marked in black color) and firstorder KF estimate (marked in red color). These results clearly show that the second-order KF-based estimator tracks the actual shadow power more accurately than the first-order KF-based estimator. Average Error for the secondorder KF-based estimator is lower than the first-order KF-based estimator. Also,

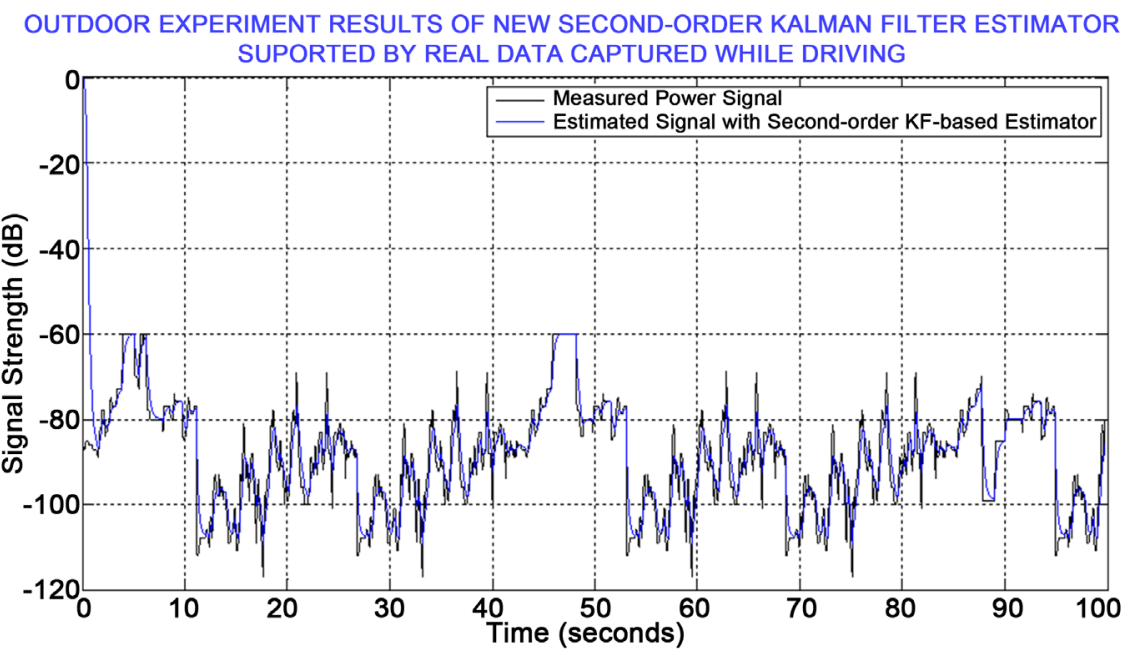

Figure 8. Power estimation in amobile station with asecond-order KF-based estimator using real data from the outdoor experiment.
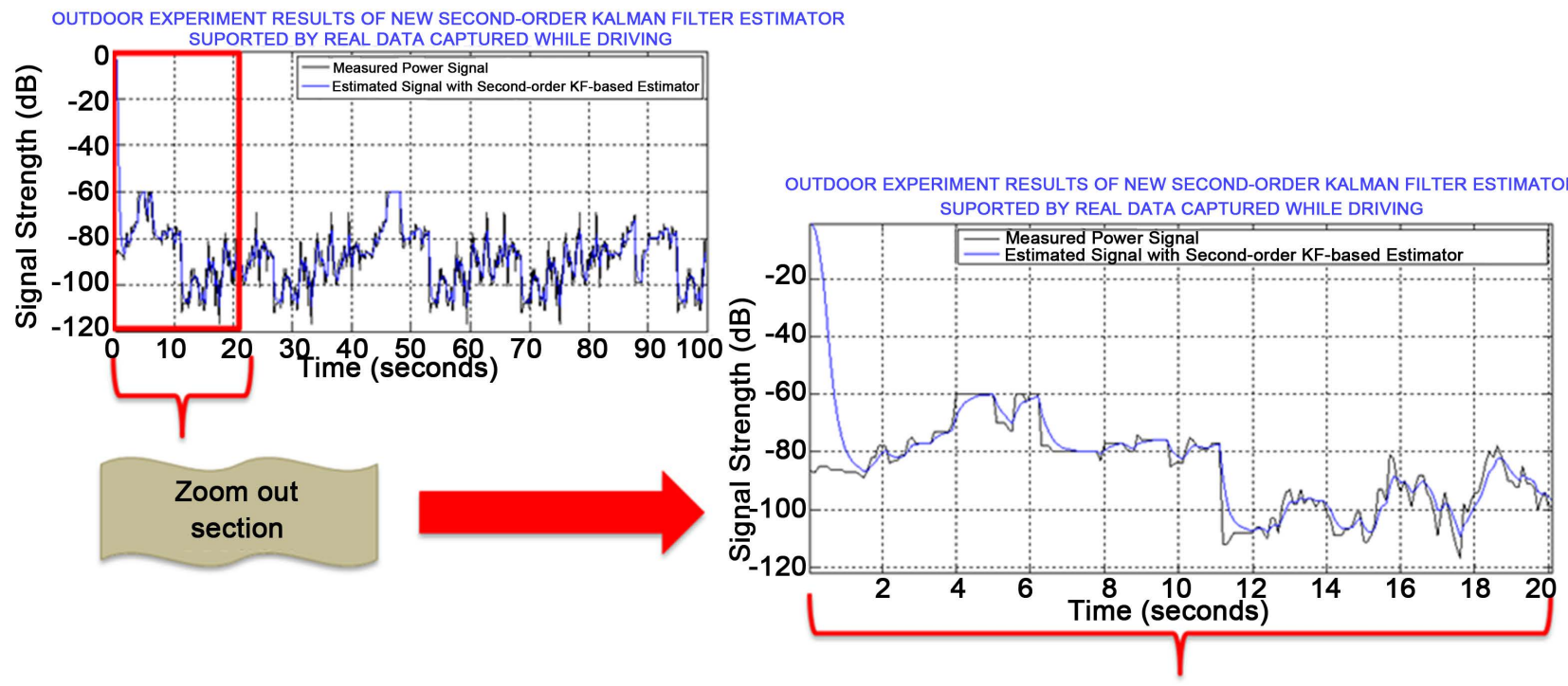

Figure 9. Zoomed out version of Figure 8 for better visualization. 


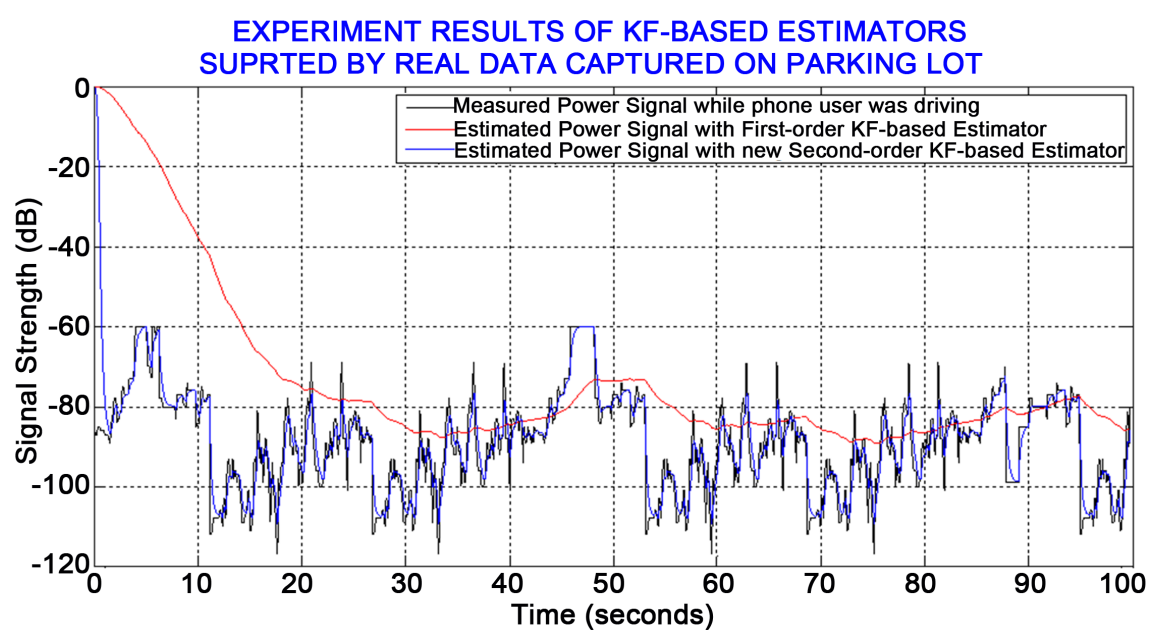

Figure 10. Comparison of system performance with the integrated second-order KF versus the same system with the first-order KF, which shows that the implementation of the second-order KF results in better estimation.

the second-order Kalman filter output has less lag from the actual shadow power.

Authors in [5] presented results demonstrating that the first-order Kalman Filter method is superior to conventional window-based estimators like the sample average estimator, the uniformly minimum variance unbiased estimator, and the maximum likelihood estimator. Our results show that the second-order KF-based estimator improves the signal estimate significantly over the first-order KF estimate.

\section{Conclusions}

In this work, a second-order KF-based estimator has been further investigated in the outdoor environment, which is able to estimate local mean shadow power in mobile communications corrupted by multipath noise. In our experiments, we mainly explored how the second-order KF-based estimator compares to the first-order KF-based estimator. Based on our results from the indoor experiments of small-scale fading presented in [2], we concluded that the second-order KF-based estimator is more accurate in predicting local mean (shadow) power profiles than the first-order KF-based estimator, even in channels with imposed non-Gaussian measurement noise. To fully complete the proposed theory, we recently extended the research to the outdoor environment and compared how these two estimators handled variability due to higher vehicle speed, larger distances, and distant large objects in the outdoor environment, such as mountains or large buildings. A Telecom tool/software released to market in 2017 was used to measure cell phone signal strength and other key parameters outside of the lab environment. Signal measurements have been conducted in typical environments like urban and suburban areas.

In this paper, we presented results from outdoor experiments which further confirmed validation of the proposed method and the theoretical analysis. The 
results supported by field data are provided in Figures 8-10. These plots clearly show that the second-order Kalman filter tracks the actual shadow power more accurately than the first-order Kalman filter. The system was able to operate without a failure under variety of conditions, which demonstrates model robustness. With MATLAB software, we were able to efficiently explore, analyze, and visualize measured data from the outdoor experiment. Comparison analysis was performed as explained in [11] [12]. Simulations in the MATLAB environment, laboratory, and outdoor experiment results have been consistent in showing that our implementation of the second-order KF results in better estimation.

\section{Future Work}

Math Works currently offers some basic examples of Kalman Filter theory. Therefore, we will most likely share our code for a first-order KF-based estimator and second-order KF-based estimator by deploying an Application with MATLAB, so others can use it too. According to MathWorks' web site, there is a wide range of options for deploying and sharing an application that was developed in MATLAB. As future work, we will look into these options.

When the channel is nonlinear, the Unscented Kalman filter also can be applied to the state space model to further improve and optimize the shadow power presented in this paper. The Unscented Kalman filter is popular due to its superiority in approximating and estimating nonlinear systems and its ability to handle non-Gaussian noise environments [13]. We may consider this optimization in the future.

As future work, we also are considering designing a third-order KF-based estimator. When the order of the filter is higher, we predict that there will be better noise repair. However, there is a tradeoff between three things: order of filter, computational difficulty of filter, and accuracy. Therefore, we need to look at these to determine if higher order estimators are practical.

\section{Acknowledgements}

The authors would like to express appreciation to Eric Yaharmatter from Autoliv Inc. for his initial thoughts on this subject.

\section{Conflict of Interest}

The authors declare that they have no conflict of interest.

\section{References}

[1] Mawari, R., Henderson, A., Akbar, M., Dargin, G. and Zohdy, M. (2016) ) An Improved Characterization of Small Scale Fading Based on 2D Measurements and Modeling of a Moving Receiver in an Indoor Environment. Journal of Signal and Information Processing, 7, 160-174. https://doi.org/10.4236/jsip.2016.73016

[2] Kapetanovic, A., Mawari, R. and Zohdy, M. (2016) Second-Order Kalman Filtering Application to Fading Channels Supported by Real Data. Journal of Signal and In- 
formation Processing, 7, 61-74. https://doi.org/10.4236/jsip.2016.72008

[3] Graf, Z. (1974) Dictionary of Electronics.

[4] Yarhmatter, E. and Kapetanovic, A. (2012) Power Estimation in Mobile Communications: Comparison of the First Order AR Model to Second Order AR Model. Oakland University, Rochester, MI. (Unpublished)

[5] Jiang, T., Sidiropoulos, N.D. and Giannakis, G.B. (2003) Kalman Filtering for Power Estimation in Mobile Communications, IEEE Transactions on Wireless Communications, 2,151-161. https://doi.org/10.1109/TWC.2002.806386

[6] Kalman, R.E. (1960) A New Approach to Linear Filtering and Prediction Problems. Research Institute for Advanced Study, Baltimore.

[7] Simon, D. (2006) Optimal State Estimation: Kalman, $\mathrm{H} \infty$ and Nonlinear Approaches. 1st Edition, John Wiley \& Sons Inc., New Jersey. https://doi.org/10.1002/0470045345

[8] Nsour, A., Abdallah, A.-S. and Zohdy, M. (2013) An Investigation into Using Kalman Filtering for Phase Estimation in Bluetooth Receivers for Gaussian and Non-Gaussian Noise. 2013 IEEE International Conference on Electro/Information Technology, Rapid City, 9-11 May 2013, 1-5. https://doi.org/10.1109/EIT.2013.6632644

[9] Rappaport, T.S. (2010) Wireless Communications Principles and Practice. $2^{\text {nd }}$ Edition, Persons Education, London.

[10] Gudmundson, M. (1991) Correlation Model for Shadowing Fading in Mobile Radio Systems. Electronics Letters, 27, 2145-2146. https://doi.org/10.1049/el:19911328

[11] Grewal, M.S. and Andrews, A.P. (2014) Kalman Filtering Theory and Practice Using MATLAB. 4th Edition, John Wiley \& Sons Inc., New York.

[12] Brown, R.G. and Hwang, P.Y.C. (2012) Introduction to Random Signals and Applied Kalman Filtering with Matlab Exercises. 4th Edition, John Wiley \& Sons Inc., Hoboken.

[13] Li, L. and Xia, Y. (2013) Unscented Kalman Filter over Unreliable Communication Networks with Markovian Packet Dropouts. IEEE Transactions of Automatic Control, 58, 3224-3230. https://doi.org/10.1109/TAC.2013.2263650

Submit or recommend next manuscript to SCIRP and we will provide best service for you:

Accepting pre-submission inquiries through Email, Facebook, LinkedIn, Twitter, etc. A wide selection of journals (inclusive of 9 subjects, more than 200 journals)

Providing 24-hour high-quality service

User-friendly online submission system

Fair and swift peer-review system

Efficient typesetting and proofreading procedure

Display of the result of downloads and visits, as well as the number of cited articles

Maximum dissemination of your research work

Submit your manuscript at: http://papersubmission.scirp.org/

Or contact jsip@scirp.org 